\title{
Switched Positive Linear Systems
}

\author{
Franco Blanchini \\ University of Udine \\ blanchini@uniud.it \\ Patrizio Colaneri \\ DEIB-Politecnico di Milano and IEIIT-CNR \\ colaneri@elet.polimi.it \\ Maria Elena Valcher \\ University of Padova \\ meme@dei.unipd.it
}



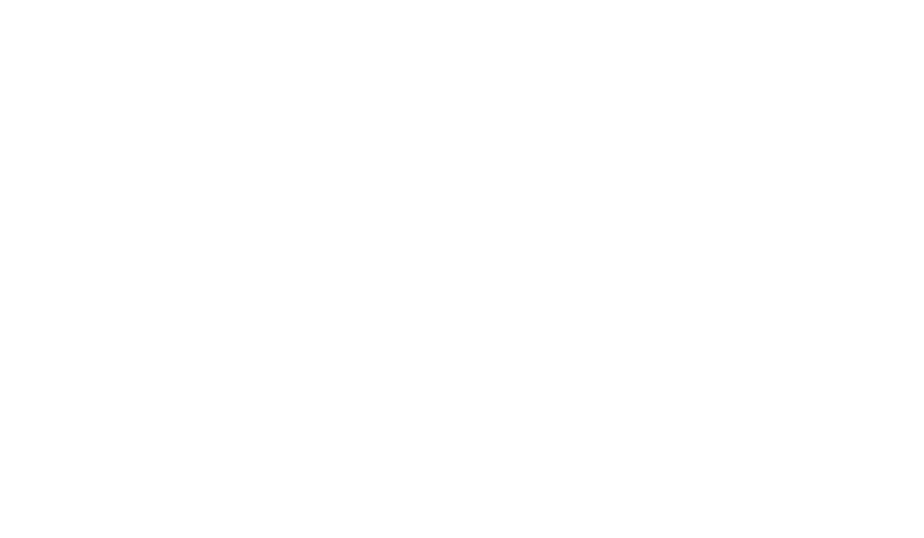

\section{Abstract}

In this monograph we consider the class of continuous-time positive switched systems. We discuss several problems, including stability, performance analysis, stabilization via switching control, and optimization. The monograph starts with a chapter where several application examples are provided, to motivate the interest in this class of systems. The rest of the monograph is dedicated to the theory of stability, stabilization and performance optimization of positive switched systems. The main existing results are recalled, but also new challenging problems are proposed and solved. Special attention has been devoted to point out those results that specifically pertain to positive (linear) switched systems and do not find a counterpart for the wider class of (nonpositive) linear switched systems. 


\section{1 \\ Introduction}

Positive systems are an important class of systems that frequently arise in application areas, such as in the chemical process industry, electronic circuit design, communication networks and biology.

Stability problems arising in the study of positive systems differ from those pertaining to standard systems. The main difference stems from the fact that the state variables are confined to the positive orthant. Thus, the whole analysis of these systems focuses only on the trajectories generated under positivity constraints, and consequently stability can be deduced from the existence of copositive Lyapunov functions whose derivatives are required to be negative only along the system trajectories in the positive orthant.

Switched positive systems also arise in a variety of applications. Examples can be found in TCP congestion control, in processes described by non-homogeneous Markov chains, in image processing, in biochemical networks, etc... Differently from general switched systems, that have received a lot of attention in the past years, the theory for positive switched systems is still in a relative infancy.

In this monograph we study the stability, performance evaluation, stabilization via switching control and optimal control of (continuous- 
time and linear) positive switched systems. We discuss results that have already been established in the literature, but other results, especially those regarding norm computation and optimization, are new and integrated with the previous ones.

In Chapter 2 we present many examples and motivations for studying positive switched systems. These examples include thermal systems, fluid networks, traffic systems, biological and epidemiological models and transmission networks. We present some specific problems that should be inspirational (at least we hope they are) for the subsequent chapters.

In Chapter 3, we consider the stability problem, namely the problem of determining stability under arbitrary switching. We show that this problem can be generalized to the problem of establishing a convergence (or divergence) rate. We characterize the stability property in terms of the existence of convex homogeneous Lyapunov functions. In general these functions can be extremely complex, so we provide some special classes of Lyapunov functions, including copositive linear and copositive quadratic Lyapunov functions, which are conservative but simpler to be computed. We also discuss a famous conjecture, now disproved in the general case, regarding the equivalence between stability under arbitrary switching and Hurwitz robustness, namely the fact that all the matrices in the convex hull of the family of system matrices are Hurwitz. The statement is true for 2-dimensional systems and false in general, since Hurwitz robustness is only necessary when the system dimension $n$ is greater than 2 . We also investigate the case when dwell time is imposed on the switching signals, namely a minimum amount of time has to elapse between any pair of consecutive switching times.

In Chapter 4 we discuss the performance evaluation of positive switched systems in terms of several input-output induced norms. Notwithstanding the fact that, for positive systems, it is often easy to establish the worst-case signal, namely the one providing the largest output norm, computing these norms is in general hard. Then we provide computationally tractable ways to generate upper bounds, for both arbitrary switching signals and dwell-time constrained ones. 
In Chapter 5 we consider the stabilization problem for systems for which the switching signal represents a control input. This problem has some interesting properties that are the counterpart of some properties established in the stability analysis case. Stabilizability is equivalent to the existence of a concave homogeneous copositive control Lyapunov function. Again, finding any such function is in general hard, so we investigate special classes including linear and quadratic copositive functions. We also provide some sufficient stabilizability conditions in terms of Lyapunov Metzler inequalities. The disproved conjecture about stability has a counterpart for the stabilizabity case: is stabilizability equivalent to the existence of at least one Hurwitz element in the convex hull of the matrices? Again the is true for 2-dimensional systems and false in general, as the existence of a Hurwitz convex combination is only a sufficient condition for stabilization. It is interesting to note that the existence of a Hurwitz convex combination is a necessary and sufficient condition for the existence of a smooth homogeneous control Lyapunov function.

Finally, in Chapter 6, we consider the optimal control problem for positive systems with a controlled switching signal. We show how some of the material presented in the previous chapters, such as the Lyapunov Metzler inequalities technique, can be successfully exploited to derive some conditions that allow to design a guaranteed cost control.

In addition to the simple numerical examples provided in Chapters 3-5 to illustrate the developed theory, in this chapter simulations are provided for a couple of "realistic" examples presented in Chapter 2, dedicated to the motivational part, and specifically: the optimal therapy scheduling for mitigation of the HIV viral load, and the disease free control applied to a SIS (Susceptible-Infective-Susceptible) epidemiological system.

This survey does not aim at providing an exhaustive account of all the research problems investigated in the literature and concerned with positive switched systems. Important issues have been omitted here, due to page constraints. Among them, it is worth quoting the following ones: controllability/reachability (see Fornasini and Valcher 2011, Santesso and Valcher 2008, Valcher and Santesso [2010, Valcher 
2009, Xie and Wang 2006]), observability of positive switched systems (Li et al. [2014]), positive switched systems with delays (Li et al. 2013a, b, Liu and Dang 2011, Liu and Lam 2012, Xiang and Xiang 2013), and interesting characterizations like joint spectral properties and asymptotic properties of matrix semigroups (Guglielmi and Protasov 2013], Protasov et al. [2010], Jungers 2012, and extremal norms for linear inclusions, Mason and Wirth 2014]). For all these topics we refer the interested Reader to the previous references. On the other hand, for the topics specifically addressed in this monograph, no references are provided in this introduction, being them appropriately quoted when needed within the text.

\subsection{Notation}

The notation used throughout the monograph is standard for positive systems. The sets of real and natural numbers are denoted by $\mathbb{R}$ and $\mathbb{N}$, respectively, while $\mathbb{R}_{+}$is the set of nonnegative real numbers. Capital letters denote matrices, small (bold face) letters denote vectors. For matrices or vectors, $\left({ }^{\top}\right)$ indicates transpose. The $(\ell, j)$ th entry of a matrix $A$ is denoted by $[A]_{\ell, j}$, while the $i$ th entry of a vector $\mathbf{x}$ is $x_{i}$ or $[\mathbf{x}]_{i}$. When the vector $\mathbf{x}$ is obtained as the result of some mathematical operation, e.g. $\mathbf{x}=A \mathbf{y}$, we will generally adopt the latter notation $[A \mathbf{y}]_{i}$. The symbol $\mathbf{e}_{i}$ denotes the $i$ th canonical vector in $\mathbb{R}^{n}$, where $n$ is always clear from the context, while $\mathbf{1}_{n}$ denotes the $n$-dimensional vector with all entries equal to 1 . The symbol $I_{n}$ denotes the identity matrix of order $n$.

A (column or row) vector $\mathbf{x} \in \mathbb{R}^{n}$ is said to be nonnegative, $\mathbf{x} \geq 0$, if all its entries $x_{i}, i=1,2, \ldots, n$, are nonnegative. It is positive if nonnegative and at least one entry is positive. In this case, we will use $\mathbf{x}>0$. It is said to be strictly positive if all its entries are greater than 0 , and in this case, we will use the notation $\mathbf{x} \gg 0$. The set of all $n$ dimensional nonnegative vectors is denoted by $\mathbb{R}_{+}^{n}$ and referred to as the positive orthant. The expressions $\mathbf{x} \gg \mathbf{y}, \mathbf{x}>\mathbf{y}$ and $\mathbf{x} \geq \mathbf{y}$ mean that the difference $\mathbf{x}-\mathbf{y}$ is strictly positive, positive and nonnegative, respectively. Similar notation is used for the (real) matrices. 
The set of $n$-dimensional nonnegative vectors whose entries sum up to 1 is the simplex

$$
\mathcal{A}_{n}:=\left\{\alpha=\left(\alpha_{1}, \ldots, \alpha_{n}\right) \in \mathbb{R}_{+}^{n}: \sum_{i=1}^{n} \alpha_{i}=1\right\} .
$$

A square matrix $A \in \mathbb{R}^{n \times n}$ is said to be Metzler ${ }^{1}$ if its off-diagonal entries $[A]_{i j}, i \neq j$, are nonnegative. Every Metzler matrix $A$ has a real dominant eigenvalue $\lambda_{F} \in \sigma(A)$ satisfying $\operatorname{Re}\left(\lambda_{F}\right)>\operatorname{Re}(\lambda)$ for every $\lambda \in \sigma(A), \lambda \neq \lambda_{F} . \lambda_{F}$ is called the Frobenius eigenvalue of $A$, see Farina and Rinaldi 2000]. Also, associated with $\lambda_{F}$ there is always both a left and a right positive eigenvector, known as (left/right) Frobenius eigenvectors.

An $n \times n$ Metzler matrix $A$ is reducible if there exists a permutation matrix $\Pi$ such that

$$
\Pi^{\top} A \Pi=\left[\begin{array}{cc}
A_{11} & A_{12} \\
0 & A_{22}
\end{array}\right],
$$

where $A_{11}$ and $A_{22}$ are square (nonvacuous) matrices, otherwise it is irreducible. It follows that $1 \times 1$ matrices are always irreducible.

A linear state space model described by the linear differential equation $\dot{\mathbf{x}}(t)=A \mathbf{x}(t)$, where $A$ is a Metzler matrix, is called a positive system, see Berman et al. 1989, Farina and Rinaldi 2000, Kaczorek 2002, Krasnoselskii 1964, Luenberger 1979], because it enjoys the property that any trajectory starting in the positive orthant remains confined in it.

A square matrix is Hurwitz if all its eigenvalues lie in the open left half plane. A Metzler matrix is Hurwitz if and only if there exists a vector $\mathbf{v} \gg 0$ such that $\mathbf{v}^{\top} A \ll 0$, or, equivalently if and only there exists a vector $\mathbf{w} \gg 0$ such that $A \mathbf{w} \ll 0$, see e.g. Farina and Rinaldi 2000 .

Given two matrices $A \in \mathbb{R}^{n \times m}$ and $B \in \mathbb{R}^{p \times q}$, the expression $C=$ $A \otimes B \in \mathbb{R}^{n p \times m q}$ stands for the usual Kronecker product. If $A \in \mathbb{R}^{n \times n}$ and $B \in \mathbb{R}^{p \times p}$, their Kronecker sum is defined as $A \oplus B=A \otimes I_{p}+$

\footnotetext{
${ }^{1} \mathrm{~A}$ Metzler matrix is also known in the literature as "essentially nonnegative matrix" (see Berman et al. [1989, Horn and Johnson [1985]) or as the opposite of a "Z-matrix" (see Horn and Johnson [1991]).
} 
$I_{n} \otimes B \in \mathbb{R}^{n p \times n p}$. Properties of Kronecker operators can be found in Graham 1981.

The symbols $\succ, \succeq, \prec$ and $\preceq$ are used to denote order relations induced by definiteness properties. For instance, the expression $P=$ $P^{\top} \succ 0 \in \mathbb{R}^{n \times n}$ means that $P$ is a (symmetric and) positive definite matrix, i.e. $\mathbf{x}^{\top} P \mathbf{x}>0$ for every $\mathbf{x} \neq 0 . P_{1} \succeq P_{2}$ means that $P_{1}-P_{2}$ is a (symmetric and) positive semi-definite matrix.

\subsection{Continuous-time positive switched systems}

A continuous-time positive switched system is described by the following equation

$$
\dot{\mathbf{x}}(t)=A_{\sigma(t)} \mathbf{x}(t), \quad t \in \mathbb{R}_{+},
$$

where $\mathbf{x}(t)$ denotes the value of the $n$-dimensional state variable at time $t$, and $\sigma(t)$ is a right-continuous and piece-wise constant mapping from $\mathbb{R}_{+}$into the finite set $\{1, \ldots, M\}$. This latter property ensures that in any bounded time interval the map $\sigma$ has always a finite number of discontinuities, known as switching instants and denoted in the following by $0=t_{0}<t_{1}<t_{2}<\ldots$. This amounts to saying that $\sigma(t)$ takes some constant value $i_{k} \in\{1,2, \ldots, M\}$ at every $t \in\left[t_{k}, t_{k+1}\right)$ and that $\sigma\left(t_{k}\right) \neq \sigma\left(t_{k+1}\right)$. In the sequel, when we will refer to an "arbitrary switching signal" $\sigma$ we will always mean an arbitrary switching signal endowed with the aforementioned properties and we will denote the set of such switching signals by the symbol $\mathcal{D}_{0}$. The reason for this notation will be clarified later on.

A function $\mathrm{x}: \mathbb{R}_{+} \mapsto \mathbb{R}^{n}$ is a solution of (1.1) if, see Shorten et al. 2007, it is continuous and piecewise continuously differentiable and if there is a switching signal $\sigma$ such that (1.1) holds at every $t \in \mathbb{R}_{+}$, except at the switching instants. For every value $i$ taken by the switching signal $\sigma$ (at $t$ ), $\dot{\mathbf{x}}(t)=A_{i} \mathbf{x}(t)$ is a (autonomous $\mathrm{2}^{2}$ continuous-time positive system, which means that $A_{i}$ is an $n \times n$ Metzler matrix. This ensures that if $\mathbf{x}(0)$ belongs to the positive orthant $\mathbb{R}_{+}^{n}$, then, for every choice of $\sigma$, the state evolution $\mathbf{x}(t)=\mathbf{x}(t ; \mathbf{x}(0), \sigma)$ belongs to $\mathbb{R}_{+}^{n}$ for

\footnotetext{
${ }^{2}$ In this monograph by an autonomous system we will always mean a system with no inputs, see Khalil 2002, Sun and Ge 2005, Willems 1970.
} 
every $t \in \mathbb{R}_{+}$. It is worth noticing that also for switched systems the Metzler property of the matrices $A_{i}, i \in\{1,2, \ldots, M\}$, is both necessary and sufficient to ensure that all the state trajectories starting in the positive orthant remain in $\mathbb{R}_{+}^{n}$ at all subsequent times, for every choice of the switching signal. Given any initial state $\mathbf{x}_{i} \in \mathbb{R}_{+}^{n}$, any switching signal $\sigma: \mathbb{R}_{+} \mapsto\{1,2, \ldots, M\}$, and any pair of time instants $t \geq \tau \geq 0$, the state at time $t$ can be expressed as

$$
\mathbf{x}(t)=\Phi(t, \tau, \sigma) \mathbf{x}_{i},
$$

where $\Phi(t, \tau, \sigma)$ represents the state transition matrix of system (1.1) corresponding to the time interval $[\tau, t]$ and the switching signal $\sigma$. Clearly, if we denote by $\tau=t_{1}<t_{2}<\cdots<t_{k}<t_{k+1}=t$ the switching instants in the time interval $[\tau, t]$ and by $i_{h}$ the value of the switching signal $\sigma$ in the time interval $\left[t_{h}, t_{h+1}\right), h \in\{1,2, \ldots, k\}$, then

$$
\Phi(t, \tau, \sigma)=e^{A_{i_{k}}\left(t-t_{k}\right)} \ldots e^{A_{i_{2}}\left(t_{3}-t_{2}\right)} e^{A_{i_{1}}\left(t_{2}-\tau\right)} .
$$

In the following, we will also consider non-autonomous positive switched systems, described, for instance (but not only), by the following equations:

$$
\begin{aligned}
& \dot{\mathbf{x}}(t)=A_{\sigma(t)} \mathbf{x}(t)+B_{\sigma(t)} \mathbf{u}(t), \\
& \mathbf{y}(t)=C_{\sigma(t)} \mathbf{x}(t)+D_{\sigma(t)} \mathbf{u}(t), \quad t \in \mathbb{R}_{+},
\end{aligned}
$$

where $\mathbf{x}(t), \mathbf{u}(t)$ and $\mathbf{y}(t)$ are the $n$-dimensional state variable, the $m$ dimensional input variable and the $p$-dimensional output variable, respectively, at time $t$. For every value $i$ taken by $\sigma$ (at $t$ ), $A_{i}$ is an $n \times n$ Metzler matrix, while $B_{i}, C_{i}$ and $D_{i}$ are nonnegative matrices. Under these conditions, the nonnegativity of the input at every time $t \geq 0$ and the nonnegativity of the initial condition $\mathbf{x}(0)$ ensure the nonnegativity of the state and output trajectories at every $t \geq 0$. 


\section{References}

A. A. Agrachev and D. Liberzon. Lie-algebraic stability criteria for switched systems. SIAM Journal on Control and Optimization, 40(1):253-269, 2001.

AIDSinfo. Panel of antiretroviral guidelines for adults and adolescents, "guidelines for the use of antiretroviral agents in HIV-1 infected adults and adolescents, 2011", 2011.

http://www.aidsinfo.nih.gov.

M. Ait Rami, V. S. Bokharaie, O. Mason, and F. R. Wirth. Stability criteria for sis epidemiological models under switching policies. Discrete and continuous dynamical series B, 19:2865-2887, 2014.

M. Akar, A. Paul, M.G. Safonov, and U. Mitra. Conditions on the stability of a class of second order switched systems. IEEE Transactions on Automatic Control, 51:338-340, 2006.

C. D. Aliprantis and R. Tourky. Cones and duality. American Mathematical Society, 2007.

L. Allherand and U. Shaked. Robust stability and stabilization of linear switched systems with dwell time. IEEE Transactions on Automatic Control, 56(2):381-386, 2011.

M. Araki. Application of M-matrices to the stability problems of composite dynamical systems. Journal of Mathematical Analysis and Applications, 52 (32):309-321, 1975.

Z. Artstein. Stabilization with relaxed controls. Nonlinear Anal., 7(11):11631173, 1983. 
J. P. Aubin. Viability theory. Systems \& Control: Foundations \& Applications. Birkhäuser Boston Inc., Boston, MA, 1991. ISBN 0-8176-3571-8.

J. P. Aubin and A. Cellina. Differential inclusions; Set Valued Maps and Viability Theory. Springer-Verlag, 1984.

X. Bai and X. S. Yang. A new proof of a theorem on optimal control of switched systems. Journal of Mathematical Analysis and Applications, 331 (2):895-901, 2007.

N. E. Barabanov. Absolute characteristic exponent of a class of linear nonstationary systems of differential equations. Siberian Mathematical Journal, 29:521-530, 1988.

N. E. Barabanov. On the Aizerman problem for third-order time-dependent systems. Differential Equations, 29:1439-1448, 1993.

M. Bardi and I. Capuzzo-Dolcetta. Optimal control and viscosity solutions of Hamilton-Jacobi-Bellman equations. Birkhäuser Boston, 2008.

S. C. Bengea and R. A. DeCarlo. Optimal control of switching systems. Automatica, 41(1):11-27, 2005.

A. Berman, M. Neumann, and R. J. Stern. Nonnegative matrices in dynamic systems. J.Wiley \& Sons, NY, 1989.

S. Bittanti and P. Colaneri. Periodic Systems: Filtering and Control. Communications and Control Engineering. Springer, 2009.

F. Blanchini. Set invariance in control: a survey. Automatica, 35:1747-1767, 1999.

F. Blanchini and P. Colaneri. Vertex/plane characterization of the dwell-time property for switched linear systems. In Proceedings of the IEEE Conference on Decision and Control, Baltimore, 2010.

F. Blanchini and G. Giordano. Piecewise-linear Lyapunov functions for structural stability of biochemical networks. Automatica, 50:2482-2494, 2014.

F. Blanchini and S. Miani. A universal class of smooth functions for robust control. IEEE Transaction on Automatic Control, 44(3):641-647, 1999.

F. Blanchini and S. Miani. Stabilization of lpv systems: state feedback, state estimation and duality. SIAM Journal on Control and Optimization, 32(1): 76-97, 2003.

F. Blanchini and S. Miani. Set theoretic methods in control. Birkhäuser, Berlin, 2008. 
F. Blanchini and C. Savorgnan. Stabilizability of switched linear systems does not imply the existence of convex Lyapunov functions. Automatica, 44(4): 1166-1170, 2008.

F. Blanchini, P. Colaneri, and M. E. Valcher. Co-positive Lyapunov functions for the stabilization of positive switched systems. IEEE Transactions on Automatic Control, AC-57:3038-3050, 2012.

F. Blanchini, P. Colaneri, and M.E. Valcher. Co-positive Lyapunov functions for the stabilization of positive switched systems. In Proceedings of the 45th Conference on Decision and Control. IEEE, 2013.

F. Blanchini, P. Colaneri, and R. H. Middleton. A convexity result for the optimal control of a class of positive nonlinear systems. In Proceedings of the IFAC World Congress, Cape Town, South Africa, 2014.

V. D. Blondel, J. Theys, and A. A. Vladimirov. An elementary counterexample to the finiteness conjecture. SIAM Journal on Matrix Analysis and Applications, 24:963-970, 2003.

P. Bolzern, P. Colaneri, and G. De Nicolao. Stochastic stability of positive Markov jump linear systems. Automatica, 50(4):1181-1187, 2014.

I. M. Bomze. Copositive optimization- recent developments and applications. European Journal of Operational Research, 216 (3):509-520, 2012.

U. Boscain. Stability of planar switched systems: the linear single input case. SIAM Journal on Control and Optimization, 41(1):89-112, 2002.

T. Bousch and J. Mairesse. Asymptotic height optimization for topical IFS, Tetris heaps, and the finiteness conjecture. Journal of the American Mathematical Society, 15:77-111, 2001.

P. Brandi and A. Salvadori. On measure differential inclusions in optimal control theory. Rendiconto Seminario Matematico Università Pol. Torino, page 69, 1998.

M. S. Branicky. Multiple Lyapunov functions and other analysis tools for switched and hybrid systems. IEEE Transactions on Automatic Control, 43:475-482, 2007.

R. K. Brayton and C. H. Tong. Constructive stability and asymptotic stability of dynamical systems. IEEE Transactions on Circuits and Systems, 27(11): 1121-1130, April 1980.

A. Bressan and B. Piccoli. Introduction to the mathematical theory of control. AIMS Series in Applied Mathematics, Springfield, 2007. 
C. Briat. Robust stability and stabilization of uncertain linear positive systems via integral linear constraints: $L_{1}$-gain and $L_{\infty}$-gain characterization. Int. Journal of Robust and Nonlinear Control, 23:1932-1954, 2013.

S. Bundfuss and M. Dur. An adaptive linear approximation algorithm for copositive programs. SIAM Journal on Optimization, 20 (1):30-53, 2009a.

S. Bundfuss and M. Dur. Copositive lyapunov functions for switched systems over cones. Systems \& Control Letters, 58 (5):342-345, $2009 \mathrm{~b}$.

C. G. Cassandras, D. L. Pepyne, and Y. Wardi. Optimal control of a class of hybrid systems. IEEE Transactions on Automatic Control, 46(3):398-415, 2001.

G. Chesi. Domain of Attraction: Analysis and Control via SOS Programming. Springer-Verlag, 2011.

G. Chesi, P. Colaneri, J. C. Geromel, R. H. Middleton, and R. N. Shorten. A non-conservative lmi condition for stability of switched systems with guaranteed dwell time. IEEE Transaction on Automatic Control, 57:12971302, 2012.

J. E. Cohen. Convexity of the dominant eigenvalue of an essentially nonnegative matrix. Proceedings of the American Mathematical Society, 81(4): 657-658, 1981.

P. Colaneri, R. H. Middleton, Z. Chen, D. Caporale, and F. Blanchini. Convexity of the cost functional in an optimal control problem for a class of positive switched systems. Automatica, 50:1227-1234, 2014.

I. Craig and X. Xia. Can HIV/AIDS be controlled? Applying control engineering concepts outside traditional fields. IEEE Control Systems Magazine, 25 (1):80-83, 2005.

R. M. D'Amato, R. T. D'Aquila, and L. M. Wein. Management of antiretroviral therapy for HIV infection: modelling when to change therapy. Antiviral Therapy, 3:147-158, 1998.

W. P. Dayawansa and C. F. Martin. A converse Lyapunov theorem for a class of dynamical systems which undergo switching. IEEE Transaction on Automatic Control, 44:751-760, 1999.

D. Del Vecchio and R. M. Murray. Biomolecular Feedback Systems. Princeton University Press, 2014.

A. V. Dmitruk and A. M. Kaganovich. The hybrid maximum principle is a consequence of Pontryagin maximum principle. Systems \& Control Letters, 57(11):964-970, 2008. 
A. V. Dmitruk and A. M. Kaganovich. Maximum principle for optimal control problems with intermediate constraints. Computational Mathematics and Modeling, 22(2):180-215, 2011.

F. Dörfler and F. Bullo. Synchronization in complex oscillator networks: A survey. Automatica, 50(6):1539-1564, 2014.

E. Eisele and R. Siliciano. Redefining the viral reservoirs that prevent HIV-1 eradication. Immunity, 37(3):377-88, 2012.

D. Elliott. Bilinear Control Systems: Matrices in Action. Springer, 2009.

L. Fainshil, M. Margaliot, and P. Chigansky. On the stability of positive linear switched systems under arbitrary switching laws. IEEE Transaction on Automatic Control, 54:807-889, 2009.

L. Farina and S. Rinaldi. Positive linear systems: theory and applications. SWiley-Interscience, Series on Pure and Applied Mathematics, New York, 2000 .

M. Feinberg. Chemical reaction network structure and the stability of complex isothermal reactors: The deficiency zero and deficiency one theorems. Chemical Engineering Science, 42:2229-2268, 1987.

E. Fornasini and M. E. Valcher. Linear copositive Lyapunov functions for continuous-time positive switched systems. IEEE Transaction on Automatic Control, 55:1933-1937, 2009.

E. Fornasini and M. E. Valcher. Stability and stabilizability criteria for discrete-time positive switched systems. IEEE Transaction on Automatic Control, 57:1208-1221, 2012.

E. Fornasini and M.E Valcher. Reachability of a class of discrete-time positive switched systems. SIAM Journal on Control and Optimization, 49 (1):162184, 2011.

E. Fornasini and M.E. Valcher. Stability properties of a class of positive switched systems with rank one difference. Systems and Control Letters, 64:12-19, 2014.

R. A. Freeman and P. V. Kokotović. Inverse optimality in robust stabilization. SIAM Journal on Control and Optimization, 34(4):1365-1391, 1996.

K. M. Garg. Theory of Differentiation: A Unified Theory of Differentiation Via New Derivate Theorems and New Derivatives. Wiley-Interscience, New York, 1998. 
M. Gaye, Y. Chitour, and P. Mason. Properties of Barabanov norms and extremal trajectories associated with continuous-time linear switched systems. In Proc. of the 52nd IEEE Conference on Decision and Control, pages 716-721, Firenze, Italy, 2013.

J. C. Geromel and P. Colaneri. Stability and stabilization of continuous-time switched systems. SIAM Journal on Control and Optimization, 45:19151930, 2006.

M. S. Gowda. On copositive and completely positive cones, and Ztransformations. Electronic Journal of Linear Algebra, 23:198-211, 2012.

A. Graham. Kronecker products and matrix calculus: with applications. Ellis Horwood series in mathematics and its applications. Horwood, 1981.

N. Guglielmi and V. Protasov. Exact computation of joint spectral characteristics of linear operators. Foundations of Computational Mathematics, 13(1):37-97, 2013.

L. Gurvits. Stability of discrete linear inclusions. Linear Algebra and its Applications, 231:47-85, 1995.

L. Gurvits, R. Shorten, and O. Mason. On the stability of switched positive linear systems. IEEE Transactions on Automatic Control, 52:1099-1103, 2007.

K. G. Hare, I. D. Morris, N. Sidorov, and J. Theys. An explicit counterexample to the Lagarias0 Wang finiteness conjecture. Advances in Mathematics, 226 : 4667-4701, $201 \dot{ }$.

E. A. Hernandez-Vargas, P. Colaneri, and R. H. Middleton. Discrete-time control for switched positive systems with application to mitigating viral escape. International Journal of Robust and Nonlinear Control, 21(10): 1093-1111, 2011.

E. A. Hernandez-Vargas, P. Colaneri, and R. H. Middleton. Optimal therapy scheduling for a simplified HIV infection model. Automatica, 49:2874-2880, 2013.

E.A. Hernandez-Vargas and R.H. Middleton. Modelling the three stages in HIV infection. Journal of Theoretical Biology, 320:33-40, 2014.

J. P. Hespanha and A. S. Morse. Stability of switched systems with average dwell-time. In Proceedings of the 38th IEEE Conference on Decision and Control, pages 2655-2660, 1999.

D. Hinrichsen, N. K. Son, and P. H. A. Ngoc. Stability radii of higher order positive difference systems. Systems \& Control Letters, 49(5):377-388, 2003. 
J. B Hiriart-Urruty and A. Seeger. A variational approach to copositive matrices. SIAM Review, 52(4):593-629, 2010.

M. W. Hirsch. Systems of differential equations which are competitive or cooperative: Iii. competing species. Nonlinearity, 1:1-51, 1988.

R. A. Horn and C. R. Johnson. Matrix Analysis. Cambridge Univ. Press, Cambridge (GB), 1985.

R. A. Horn and C. R. Johnson. Topics in Matrix Analysis. Cambridge University Press, Cambridge, Cambridge, UK, 1991.

Y. Huang, H. Wu, and E. P. Acosta. Hierarchical bayesian inference for HIV dynamic differential equation models incorporating multiple treatment factors. Biometrical Journal, 52(4):470-486, 2010.

J. Joo and P. Tallos. The filippov-waziewski relaxation theorm revisited. Acta Mathematica Hungarica, 83:171-177, 1999.

R. M. Jungers. On asymptotic properties of matrix semigroups with an invariant cone. Linear Algebra and its Applications, 437(5):1205-1214, 2012.

T. Kaczorek. Positive 1D and 2D systems. Springer Verlag, London, UK, 2002.

H. K. Khalil. Nonlinear Systems, 3rd Edition. Prentice Hall, 2002.

F. Knorn, O. Mason, and R. N. Shorten. On linear co-positive Lyapunov functions for sets of linear positive systems. Automatica, 45:1943-1947, 2009.

M. A. Krasnoselskii. Positive Solutions of Operator Equations. P. Noordhoff Ltd., Groningen, 1964.

L. S. Ladson. Optimization Theory for Large Scale Systems. Series of Operation Research. MacMillan, New York, 1970.

J. Lagarias and Y. Wang. The finiteness conjecture for the generalized spectral radius of a set of matrices. Linear Algebra and its Applications, 214:17-42, 1995.

S. Li, Z. Xiang, and H. R. Karimi. Stability and $l_{1}$-gain controller design for positive switched systems with mixed time-varying delays. Applied Mathematics Computation, 222(1):507-518, 2013a.

S. Li, Z. Xiang, and H. R. Karimi. Stability and $l_{1}$-gain gain control of positive switched systems with time-varying delays via delta operator approach. Mathematical Problems in Engineering, ID 515362:10, 2013b. 
S. Li, Z. Xiang, and H. R. Karimi. Positive $l_{1}$ observer design for positive switched systems. Circuits, Systems, and Signal Processing, 33 (7):20852106, 2014.

D. Liberzon. Switching in Systems and Control. Birkhauser, Boston, 2003.

X. Liu and C. Dang. Stability analysis of positive switched linear systems with delays. IEEE Transactions on Automatic Control, 56 (7):1684 - 1690, 2011.

X. Liu and J. Lam. Stability analysis of discrete-dime positive switched linear delay systems. In Proceedings of the 2012 American Control Conference, pages 5444 - 5449, Montreal, Canada, 2012.

D. G. Luenberger. Introduction to dynamical systems. J. Wiley and Sons, 1979.

R. Luo, M. J. Piovoso, J. Martinez-Picado, and R. Zurakowski. Optimal antiviral switching to minimize resistance risk in HIV therapy. PloS one, 6 (11):e27047, 2011.

R. Luo, M. J. Piovoso, J. Martinez-Picado, and R. Zurakowski. HIV model parameter estimates from interruption trial data including drug efficacy and reservoir dynamics. PloS one, 7(7):e40198, 2012.

M. Margaliot. Stability analysis of switched systems using variational principles: An introduction. Automatica, 42(12):2059-2077, 2006.

M. Margaliot. A counterexample to a conjecture of Gurvits on switched systems. IEEE Transactions on Automatic Control, 52(6):1123-1126, 2007.

O. Mason and R. N. Shorten. On the simultaneous diagonal stability of a pair of positive linear systems. Linear Algebra and its Applications, 413:13-23, 2006.

O. Mason and R. N. Shorten. On linear copositive Lyapunov functions and the stability of switched positive linear systems. IEEE Transactions on Automatic Control, 52:1346-1349, 2007.

O. Mason and F. Wirth. Extremal norms for positive linear inclusions. Linear Algebra and its Applications, 444:100-113, 2014.

D. B. McDonald. Lyapunov optimizing sliding mode control for linear systems with bounded disturbance. Applied Mathematical Sciences, 2(19):901-918, 2008.

B. Mohar. The Laplacian spectrum of graphs. Graph Theory, Combinatorics, and Applications, 2:871-898, 1991. 
A. P. Molchanov and E. S. Pyatnitskii. Lyapunov functions that define necessary and sufficient conditions for absolute stability of nonlinear nonstationary control systems. I-II-III. Automatation and Remote Control, 47: I:344-54, II: 443-51, III:620-630, 1986.

M. M. Moldovan and M. S. Gowda. On common linear/quadratic Lyapunov functions for switched linear systems. In Nonlinear Analysis and Variational Problems: In Honor of George Isac, volume 35, pages 415-429. Springer, New York, 2010.

Y. Moreno, R. Pastor-Satorras, and A. Vespignani. Epidemic outbreaks in complex heterogeneous networks. The European Physical Journal B: Condensed Matter and Complex Systems, 26:521-529, 2002.

K. G. Murty and S. N. Kabadi. Some NP-complete problems in quadratic and non- linear programming. Mathematical Programming, 39 (2):117-129, 1987.

D. A. Ouattara, M. S. J. Mhawej, and C. H. Moog. Clinical tests of therapeutical failures based on mathematical modeling of the HIV infection. IEEE Transactions on Automatic Control, 53:230-241, 2008.

A. S. Perelson and P. W. Nelson. Mathematical analysis of HIV-i: Dynamics in vivo. SIAM Review, 41(1):3-44, 1999.

E. Plischke and F. Wirth. Duality results for the joint spectral radius and transient behaviour. Linear Algebra and its Applications, 428:2368-2384, 2008.

V. Y. Protasov, R. M. Jungers, and V. D. Blondel. Joint spectral characteristics of matrices: A conic programming approach. SIAM Journal on Matrix Analysis and Applications, 31 (4):2146-2162, 2010.

H. Putter, S. H. Heisterkamp, J. M. A. Lange, and F. De Wolf. A Bayesian approach to parameter estimation in HIV dynamical models. Statistics in Medicine, 21(15):2199-2214, 2002.

E. S. Pyatnitskii. Criterion for the absolute stability of second-order nonlinear controlled systems with one nonlinear nonstationary element. Automation and Remote Control, 32:1-11, 1971.

E. S. Pyatnitskiy and L. B. Rapoport. Periodic motion and tests for absolute stability on nonlinear nonstationary systems. Automation and Remote Control, 52:1379-1387, 1991.

E. S. Pyatnitskiy and L. B. Rapoport. Criteria of asymptotic stability of differential inclusions and periodic motions of time-varying nonlinear control systems. IEEE Transactions on Circuits and Systems- Part I, 43:219-229, 1996. 
A. Rantzer. Distributed control of positive systems. In Proceedings of the 50th IEEE Conference on Decision and Control and European Control Conference, pages 6608-6611, 2011.

A. Rantzer and B. Bernhardsson. Control of convex monotone systems. In Proceedings of the 53rd IEEE Conference on Decision and Control, 2014.

L. Rapoport. Asymptotic stability and periodic motions of selector-linear differential inclusions. In F. Garofalo and L. Glielmo, editors, Robust control via variable structure and Lyapunov techniques, pages 269-285. Springer, 1996.

P. Santesso and M. E. Valcher. Monomial reachability and zero-controllability of discrete-time positive switched systems. Systems and Control Letters, 57: 340-347, 2008.

R. Shorten, F. Wirth, O. Mason, K. Wulf, and C. King. Stability criteria for switched and hybrid systems. SIAM Review, 49:454-592, 2007.

S. Smale. On the differential equations of species in competition. Journal of Mathematical Biology, pages 5-7, 1976.

H. L. Smith. Monotone Dynamical Systems: An Introduction to the Theory of Competitive and Cooperative Systems. American Mathematical Society, 2008.

N. K. Son and D. Hinrichsen. Robust stability of positive continuous-time systems. Numerical fractional analysis and Optimization, 17:649-659, 1996.

N. K. Son and D. Hinrichsen. $\mu$-analysis and robust stability of positive linear systems. Applied Mathematics and Computer Science, 8 (2):253-268, 1998.

E. Sontag. Monotone and near-monotone biochemical networks. Systems and Synthetic Biology, 1:59-87, 2007.

J. Sponsel, S. Bundfuss, and M. Dur. An improved algorithm to test copositivity. Journal of Global Optimization, 52 (3):537-551, 2012.

Z. Sun and S. S. Ge. Switched Linear Systems: Control and Design. SpringerVerlag, London, 2005.

Z. Sun and S. S. Ge. Stability Theory of Switched Dynamical Systems. Springer-Verlag, London, 2011.

V. Utkin, J. Guldner, and M. Shijun. Sliding Mode Control in Electromechanical Systems. Automation and Control Engineering. Taylor \& Francis, 1999.

M. E. Valcher. On the $k$-switching reachability sets of single-input positive switched systems. In Proceedings of the 2009 American Control Conference, pages 2379-2384, St. Louis, 2009. 
M. E. Valcher and P. Santesso. Reachability properties of single-input continuous-time positive switched systems. IEEE Transactions on Automatic Control, 55, no.5:1117-1130, 2010.

M. A. Wicks, P. Peleties, and R. A. De Carlo. Switched controller synthesis for the quadratic stabilization of a pair of unstable linear systems. European Journal of Control, 4, no. 2:140-147, 1998.

J. L. Willems. Stability theory of dynamical systems. Nelson Publ., GB, 1970.

F. Wirth. A converse theorem for linear parameter-varying systems and linear switching systems. SIAM Journal on Control and Optimization, 44:210239, 2005.

K. Wulff, J. Foy, and R. Shorten. Comments on periodic and absolute stability for switched linear systems. In Proceedings of the American Control Conference, 2003.

M. Xiang and Z. Xiang. Observer design of switched positive systems with time-varying delays. Circuits, Systems, and Signal Processing, 32(5):21712184, 2013.

G. Xie and L. Wang. On reachability and controllability of positive discretetime switched linear systems. In Proceedings of the IEEE International Conference on Systems, Man and Cybernetics, pages 4575 - 4579, Taipei, China, 2006.

A. Zappavigna, T. Charalambous, and F. Knorn. Unconditional stability of the Foschini-Miljanic algorithm. Automatica, 48:219-224, 2012. 